\title{
Converging on neurodegenerative mechanisms
}

\author{
Diverse neurodegenerative diseases share a common pathological feature, namely the accumulation of misfolded \\ proteins. However, both drug development and research need more standardization of the biomarkers for the \\ protein types involved. The bold strategy of integrating high-throughput genetic and chemical screens in yeast \\ with experiments in neurons derived from genetically modified human induced pluripotent stem cells (iPSCs) is \\ producing many significant new molecular insights into disease mechanisms.
}

A lthough there is no evidence for transmission between individuals, the cell-to-cell transmissibility of non-prion neurodegenerative protein aggregates now provides a common framework potentially unifying the fields of Alzheimer's and Parkinson's diseases as well as other age-related neurodegenerative diseases (see review, Nat. Med. 20, 130-138, 2014). The timely joint Keystone Symposia on Parkinson's disease and Alzheimer's disease on 2-7 March 2014 (http:// www.keystonesymposia.org/14Q7 and http://www.keystonesymposia. org/14Q8) recognized this convergence to harness research interest in the similarities and differences in these, the two most common age-related neurodegenerative diseases.

Despite negative results from a number of recent clinical trials of Alzheimer's disease therapies, there is much optimism that clinical trials targeted at presymptomatic individuals at higher genetic risk of disease will prove informative, provided appropriate molecular and imaging diagnostics can be properly calibrated with respect to disease progression. Standardized measures in cerebrospinal fluid of total and phosphorylated tau protein and of $\beta$-amyloid $(A \beta 1-42)$ can be used to diagnose Alzheimer's disease pathology at earlier stages where there is mild cognitive impairment, and these biomarkers provide tools to monitor the outcomes of clinical trials with anti-amyloid immunotherapy and secretase inhibitors (Nat. Rev. Neurol. 6, 131-144, 2010). In contrast, there is much less agreement among research laboratories on methods for the measurement and interpretation of measurements of $\alpha$-synuclein and $\beta$-amyloid oligomers in fluids and tissues of humans and animal model systems, resulting in a range of methodologies and biological interpretations.

Much of our current understanding in this field derives from the genes identified by hereditary mutations in familial subtypes of amyloid and Lewy body diseases, and it is encouraging to see measures to incorporate genotyping of known prevalent mutations as well as risk-associated SNPs identified by genome-wide association studies in shared bioresources. Even a little genetic data (but preferably exome, transcriptome and genome-wide SNP microarray data) will greatly improve collaboration around biobanked tissue samples and clinical data (for example, Alzheimers Dement. doi:10.1016/j.jalz.2013.06.003, 26 August 2013), bringing together researchers with overlapping interests and promoting discovery of overlapping pathways in the different diseases.
One way to make new use of disease-causing mutations is with cell reprogramming techniques. In one such study, hereditary genetic mutations conferring high risk of Parkinson's disease were combined with known environmental stressors (Sci. Trans. Med. doi:10.1126/ scitranslmed.3003985, 2012). Phenotypic readouts for the mitochondrial pathology of the disease in this model included oxidative phosphorylation and oxidative stress, as well as mitochondrial integrity and mitophagy. The phenotypic characterization of batches of neurons differentiated from patient-derived iPSCs was carried out simultaneously in multiple laboratories, demonstrating both a proliferation in technical capability and a level of controlled scrutiny unavailable when studying individual patients.

Once a disease-causing mutation has been identified in humans, the genetic networks in which the mutation has its effect can be systematically investigated via high-throughput screens and systems modeling. Good examples of this approach are the drug and genetic modifier screens for yeast phenotypes induced by $\alpha$-synuclein toxicity, including vesicle trafficking defects (Nat. Genet. 41, 308-315, 2009 and Science 342, 979-983, 2013), and the subsequent testing of a small molecule modifier of toxicity in patient-derived iPSCs (Science 342, 983-987, 2013).

Although reprogrammed human cell lines have enormous potential as models in which to study the molecular mechanisms of neurodegenerative pathogenesis, the pluripotency of the iPSC and the stochasticity of neuronal differentiation call for stringent controls at every step. Referees remind us that for successful inference it is essential to work with genetic variants for which there is genetic evidence (linkage or association) of in vivo human neurodegenerative pathogenicity. To control for the genetic background and the lineage of the derivative cells, it is considered state of the art to generate multiple isolines of iPSCs bearing a mutation and this mutation corrected to wild type, as well as isolines of wild-type iPSCs and wild-type iPSCs modified to mutant. Because iPSCs can be converted into a range of neuron types, it is recommended that measures of genomic expression be reported sufficient to demonstrate cell fate as well as the proportions of each cell type generated at each stage. As these steps are impractically expensive to carry out for every step of every experiment, sharing materials, protocols and genomic data among competing groups will encourage the adoption of best practices to establish trusted and well-characterized standard cell models. 